\title{
Relaciones interpersonales en niños y jóvenes con trastornos del espectro del autismo y discapacidad intelectual
}

\author{
Interpersonal relationships in children and \\ adolescents with autism spectrum disorders \\ and intellectual disability
}

\section{Resumen}

Las personas con discapacidad severa y profunda han sido un colectivo poco estudiado en el ámbito de la investigación y, menos aún, en las etapas de la infancia y adolescencia. Este estudio evalúa las relaciones interpersonales de una muestra de 55 personas con Trastorno del Espectro Autismo (TEA) y discapacidad intelectual (DI) entre 4 y 20 años. Para ello se utilizó la subescala de relaciones interpersonales de la Escala KidsLife (Gómez et al., en prensa) que evalúa calidad de vida. Las puntuaciones más altas se obtuvieron en los ítems relacionados con la comunicación, mientras que las más bajas se observaron en los ítems relacionados con las interacciones sociales. Además, las personas con DI profunda obtuvieron puntuaciones significativamente más bajas que aquellos con mayores niveles de funcionamiento. Se discute acerca de las implicaciones del estudio y se realizan recomendaciones específicas para mejorar las prácticas profesionales y organizacionales dirigidas a la mejora de la calidad de vida.

\section{Palabras clave}

Relaciones interpersonales, trastornos del espectro del autismo, discapacidad intelectual, calidad de vida, evaluación.

\section{Lucía Morán Suárez \\ <moranlucia@uniovi.es>}

Universidad de Oviedo

\section{Abstract}

People with severe and profound intellectual disabilities, and especially children and adolescents within these groups, have received little attention in research. This study assesses the interpersonal relationships of 55 people with Autism Spectrum Disorders (ASD) and Intellectual Disability (ID). We applied the KidsLife Scale (Gómez et al., in press) that assesses quality of life. The highest scores were obtained for those items related to communication, while the lowest ones were found for those related to social interactions. Additionally, people with profound ID obtained significantly lower scores than those with the highest levels of functioning. A discussion about the implications of the study is provided as well as some specific recommendations for bettering professional and organizational practices for the purpose of improving quality of life.

\section{Keywords}

Interpersonal relationships, autism spectrum disorders, intellectual disability, quality of life, assessment.

\section{Laura E. Gómez Sánchez <gomezlaura@uniovi.es> \\ Universidad de Oviedo}

\section{$M^{a}$ Ángeles Alcedo Rodríguez \\ <malcedo@uniovi.es> \\ Universidad de Oviedo}

Para citar:

Morán, L. et al. (201 5): "Relaciones interpersonales en niños y jóvenes con trastornos del espectro del autismo y discapacidad intelectual", Revista Española de Discapacidad, 3 (I): 77-9I.

Doi: <http://dx.doi.org/IO.5569/23405 I04.03.01.04>

Fecha de recepción: I I-OI-20I 5 Fecha de aceptación: 24-04-20I 5 


\section{Relaciones interpersonales en niños y jóvenes con trastornos del espectro del autismo y discapacidad intelectual}

Los intentos de conceptualización y medida de la calidad de vida (CV) de las personas con discapacidad intelectual (DI) han evolucionado desde una noción meramente sensibilizadora hasta convertirse en un constructo social, un área de investigación aplicada y un principio básico de prestación de servicios (Cuesta, 2009: 29; Gómez et al., 20ıоa: 454).

Una de las definiciones más conocidas y utilizadas en el ámbito de las discapacidades intelectuales y del desarrollo, es la propuesta por Schalock y Verdugo (Schalock y Verdugo, 2003, 2007, 20I2). Según esta concepción, la CV se entiende como un estado deseado de bienestar personal que: a) es multidimensional, formado por ocho dimensiones; b) tiene propiedades universales (etic) y ligadas a la cultura (emic); c) engloba componentes objetivos y subjetivos; y d) está influenciada por características personales y factores ambientales (Schalock et al., 2010:20).

Este concepto de CV se operativiza a través de dimensiones, indicadores y resultados personales, organizados en diferentes niveles. Las dimensiones básicas de CV se entienden como "un conjunto de factores que componen el bienestar personal” (Schalock y Verdugo, 2003: 34), y que se concretan en: inclusión social, autodeterminación, bienestar emocional, bienestar físico, bienestar material, derechos, desarrollo personal y relaciones interpersonales.

A su vez, estas dimensiones se definen a través de sus indicadores centrales (Schalock et. al, 2010: 20). Estos indicadores permiten la evaluación de resultados puesto que son "percepciones, conductas o condiciones específicas de las dimensiones de calidad de vida que reflejan el bienestar de la persona" (Schalock y Verdugo, 2003: 34). La evaluación de la situación personal o de las aspiraciones de la persona en estos indicadores se refleja en los resultados personales, es decir, aquellas aspiraciones definidas y valoradas personalmente (Schalock et al., 2009: 20).

En lo relativo a la evaluación del constructo de CV, la Ley de Promoción de la Autonomía Personal y Atención a las Personas en Situación de Dependencia recoge que los instrumentos que permitan la evaluación de la CV con suficientes garantías de validez y fiabilidad son, no ya recomendables, sino necesarios e indispensables (Ley de Promoción de la Autonomía Personal y Atención a las Personas en Situación de Dependencia, BOE de I 5 de diciembre de 2006: 44I 54).

Cabe destacar que los cambios que han tenido lugar en los últimos años en el ámbito de la atención a las personas con discapacidad se están restringiendo casi exclusivamente a aquellas personas que cuentan con suficientes capacidades y habilidades de comprensión y expresión que pueden contestar de un modo fiable a los autoinformes (Gómez et al., 20I4). Estos instrumentos de evaluación son considerados como la mejor forma de evaluar la $\mathrm{CV}$, sin embargo, los informes de otras personas son necesarios como información complementaria e, incluso, pueden llegar a sustituir a los autoinformes cuando no es posible obtener directamente la información de la persona (Sheldrick et al., 20I 2: 53).

Este estudio se centra en niños y jóvenes con DI y Trastornos del Espectro del Autismo (TEA), un continuo de trastornos del desarrollo neurológico que se caracterizan por déficits en la comunicación y la interacción social, así como por patrones restringidos y repetitivos de comportamiento, intereses y actividades (American Psychiatric Association, 2013: 28; Sheldrick et al., 20I 2: 53). Las puntuaciones medias de la CV en estos niños son menores en comparación con aquellos niños que presentan otras condiciones, tales como enfermedades crónicas (Kuhlthau et al., 20I0: 726) o niños sin discapacidad (Lee et al., 2008: I I 55 ). A su vez, los padres de los niños con TEA de menor edad informan de mayores puntuaciones en la CV que los padres de los niños de mayor edad. Resulta pues importante conocer los factores que 
pueden influir en el descenso de las puntuaciones al aumentar la edad del niño, de forma que permitan la puesta en marcha de estrategias de intervención eficaces (Kuhlthau et al., 20I0: 727).

Sin embargo, no sólo la CV del niño con TEA se ve comprometida, sino también la CV de sus familias. De esta forma, los familiares de niños y adolescentes con TEA presentan un mayor nivel de sobrecarga familiar, mayores posibilidades de renunciar a un trabajo, absentismo escolar, menor participación en eventos sociales y un menor uso de los recursos comunitarios, en comparación con las familias de niños que presentan otras condiciones o no presentan discapacidad (Lee et al., 2008: I I 55 ). Este decremento en las puntuaciones de CV de los niños, y de sus familias puede estar reflejando los problemas de comportamiento de muchos niños con TEA (Lee et al., 2008: I I 48). En este sentido, si bien la CV no está consistentemente relacionada con el diagnóstico de TEA o de DI, sí se asocia con las conductas estereotipadas, la reciprocidad social y la conducta adaptativa (Kuhlthau et al., 20I0: 72I), lo que sugiere que las intervenciones dirigidas a mejorar dichos comportamientos pueden mejorar la CV tanto de las personas con TEA, como de sus familias (Lee et al., 2008: I I 59).

Cabe destacar que gran parte de la literatura revisada sobre CV en las personas con TEA se centra en el uso de conceptos como el de CV relacionada con la salud (p. ej., WHOQOL Group, 2002: 3) y CV familiar (p. ej., Summers et al., 2005: 779), en los que, si bien se han llevado a cabo notables desarrollos del concepto de CV y modelos de importancia indudable, están más centrados en aspectos concretos. En este trabajo se parte de un concepto que va más allá y aborda la CV desde una perspectiva más amplia, que incluye las áreas más relevantes de la vida de una persona y propone su evaluación mediante elementos subjetivos y objetivos: la calidad de vida individual. Las dimensiones de este constructo adquieren especial relevancia a la hora de estimar el impacto del TEA (que puede no ser visible si se analizan de forma aislada los síntomas), así como a la hora de valorar la eficacia de las intervenciones.
Una de las dimensiones fundamentales de CV es la de Relaciones Interpersonales (RI), que tiene que ver con relacionarse con distintas personas, tener amigos y llevarse bien con la gente ( $\mathrm{p}$. ej., vecinos, compañeros y otros). Algunos de los indicadores más comúnmente elegidos para operativizar la son 'Comunicación', 'Relaciones familiares', 'Amigos', 'Compañeros' y 'Sociedad' (Gómez et al., en prensa).

En el caso de las personas con TEA, la alteración de la conducta social es uno de sus rasgos centrales (Tureck y Matson, 201 2: 608). Los niños con TEA emplean menos tiempo en las interacciones sociales, presentan una menor disposición a iniciar el contacto interpersonal (Deckers et al., 20I4: 449) y tienen dificultades para comprender las emociones de los demás en comparación con los niños sin TEA (Gillis et al., 2OII: 352).

Asimismo, los niños con TEA que presentan déficits sociales obtienen menores puntuaciones en desarrollo cognitivo y emocional (Gillis et al., 20I I: 352). Además, más del 50\% de las personas con TEA presentan también DI y un $40 \%$ un nivel de DI severo o profundo (Fombonne 2003 c.p. Walton e Ingersoll, 20I3: 594). Estas personas con TEA y DI muestran un menor nivel de habilidades sociales y más conductas desafiantes que las personas con similares niveles de DI sin TEA (p. ej., personas con síndrome de Down). Lo anterior pone de relieve que presentan otro tipo de necesidades que no pueden ser cubiertas por los programas diseñados para personas que tienen solamente DI. En definitiva, las dificultades en las RI hacen que esta dimensión cobre especial relevancia en la evaluación de la CV de las personas con TEA (Walton e Ingersoll, 20I 3:595).

Por todo ello, el presente trabajo tiene como objetivo evaluar las relaciones interpersonales en niños y jóvenes con TEA y DI, con el fin último de detectar las necesidades y puntos fuertes de este colectivo en esta dimensión, y guiar de este modo los apoyos, los programas y las prácticas profesionales dirigidas a mejora de la CV. Se ahondará además en el estudio de la posible influencia de variables señaladas como relevantes 
en la literatura como el género, la edad, el tipo de escolarización, el nivel de discapacidad y la presencia de problemas de comportamiento.

\section{Método}

\subsection{Participantes}

La muestra estudiada estuvo compuesta por 55 participantes con TEA que fueron evaluados por observadores pertenecientes a I9 organizaciones y entidades situadas en Andalucía (30,9\%), País Vasco (23,6\%), Comunidad Valenciana $(23,6 \%)$, Cataluña $(5,5 \%)$ y otras comunidades (Aragón, Cantabria, Castilla-La Mancha, Castilla y León, Extremadura y La Rioja) ( $6,2 \%$ ). La mayoría eran varones $(72,7 \%)$ y su rango de edad se situó entre 4 y 20 años, con una media de I 2,6 años $(D T=4,7)$.

En cuanto a la distribución de los participantes según su nivel de DI, el I, $8 \%$ presentaba un nivel leve, el $20 \%$ moderado, el $67,3 \%$ severo y el $10,9 \%$ profundo. La media del porcentaje de discapacidad fue del 70,3\%, en un rango que osciló entre $35 \%$ y $99 \%$. El nivel de dependencia en su mayoría correspondía a Grado III o Gran dependencia (67,3\%), y el resto a un Grado II o Severa (32,7\%). Respecto a la presencia de otras condiciones de la persona evaluada, un 32,7\% presentó problemas de comportamiento, un I $4,6 \%$ discapacidad física, un $\mathrm{I} 4,5 \%$ problemas de salud mental y/o trastorno emocional, un $\mathrm{I} 4,5 \%$ epilepsia, un І०,9 \% problemas de salud graves, un 9, I \% discapacidad visual, un $5,5 \%$ parálisis cerebral, un $3,6 \%$ discapacidad auditiva, un I, $8 \%$ TDAH y un I, $8 \%$ síndrome de Down.

En cuanto al tipo de escolarización, la mayoría acudían a centros de educación especial ( $80 \%$ ). Solo el I $2,7 \%$ cursaba sus estudios en centros ordinarios, y el resto lo hacía en una modalidad de educación combinada $(7,3 \%)$.

En cuanto a las personas que cumplimentaron los cuestionarios, se contó con 26 mujeres y 7 varones, entre 26 y 6I años $(M=44,7, D T=8,9)$. Respecto a la clase de relación entre el evaluador y la persona evaluada, un $54,5 \%$ fueron profesores y educadores, un $27,3 \%$ progenitores ( $16,4 \%$ madres y го,9 \% padres), un I0,9\% psicólogos, el 3,6\% orientadores escolares, un I, $8 \%$ cuidadores y un I, $8 \%$ directores de centros. El rango de edad se situó entre 4 y 20 años, con una media de 12,6 años ( $D T=$ 4,69). La duración media de la relación entre el informador principal y la persona evaluada fue de 6 años y 4 meses (el rango osciló entre 6 meses y I7 años). La frecuencia del contacto entre el informador principal y el evaluado fue, en un $92,7 \%$ de los casos, diaria o varias veces por semana, en un I, $8 \%$ una vez a la semana y en un $5,5 \%$ una vez cada dos semanas. En cuanto al número de personas que el informador principal necesitó consultar para completar la escala, osciló entre I y 4 .

\subsection{Instrumentos de medida}

El instrumento utilizado ha sido la subescala de RI de la escala KidsLife (Gómez et al., en prensa). La escala KidsLife evalúa resultados personales relacionados con la CV de niños y adolescentes con DI menores de $2 \mathrm{I}$ años (siempre que se encuentren en el sistema educativo). Puede ser completada por un observador externo que conozca bien a la persona (al menos desde hace 6 meses) y que tenga oportunidades de observarla durante largos periodos de tiempo en diferentes entornos. Por tanto, los respondientes más idóneos suelen ser familiares, profesores y profesionales proveedores apoyos.

La versión piloto de la escala KidsLife cuenta con I 56 ítems, distribuidos en ocho subescalas que se corresponden con las ocho dimensiones de CV (i.e., inclusión social, autodeterminación, bienestar emocional, bienestar físico, bienestar material, derechos, desarrollo personal y relaciones interpersonales). El formato de respuesta presenta cuatro opciones (i.e., nunca, algunas veces, frecuentemente y siempre). Todos ellos son ítems observables, concretos y fáciles de comprender. La escala también contiene una 
presentación de los objetivos de evaluación, una declaración de consentimiento informado, un apartado de datos sociodemográficos de la persona evaluada, del informador principal y de la organización o entidad en la que la persona recibe los servicios o apoyos, una apartado que recoge la valoración general del grado de satisfacción que la persona presenta con su vida tanto antes (grado de satisfacción inicial) como después de la cumplimentación de la escala (grado de satisfacción final) y, finalmente, un apartado para añadir cualquier otra información o sugerencia. Puesto que la escala KidsLife se halla en proceso de validación, no se cuenta aún con datos sobre las propiedades psicométricas del instrumento ni con baremos que permitan interpretar las puntuaciones.

En este estudio se ha utilizado la subescala de RI que cuenta con 20 ítems de valencia positiva. Con el fin de proporcionar evidencias de fiabilidad y validez de la escala en la muestra de estudio, en primer lugar se analizó la consistencia interna de los items mediante tres procedimientos: alpha de Cronbach $(\alpha=, 879)$, la división en dos mitades de Spearman-Brown $\left(r_{x x}=, 829\right)$ y los coeficientes lambda de Guttman $\left(\lambda_{1}=, 835, \lambda_{2}=, 886\right.$ y $\left.\lambda_{3}=, 879\right)$.

En segundo lugar, se analizó la fiabilidad de los items mediante el indice de fiabilidad y el indice de dificultad. Estos índices se representan en la Tabla I. La primera columna de la tabla representa la media del ítem, que se corresponde con el índice de dificultad (ID). La tercera columna representa el índice de fiabilidad $(I F)$, equivalente al producto resultante de la multiplicación de la $D T$ por el índice de homogeneidad corregida del ítem $\left(I H_{c}\right)$. Finalmente, la cuarta columna contiene los valores de alfa corregidos.

Tabla 1. Estadísticos de los ítems de la dimensión RI

\begin{tabular}{|l|c|c|c|c|}
\hline Ítems & M(ID) & DT & IHc & ac \\
\hline RI_01 & 3,18 & 0,772 & 0,334 & 0,878 \\
\hline RI_02 & 3,36 & 0,778 & 0,6 & 0,87 \\
\hline RI_03 & 3,18 & 0,772 & 0,479 & 0,873 \\
\hline RI_04 & 2,25 & 1,075 & 0,225 & 0,885 \\
\hline RI_05 & 2,25 & 0,907 & 0,442 & 0,875 \\
\hline RI_06 & 3,16 & 0,811 & 0,451 & 0,874 \\
\hline RI_07 & 3,67 & 0,546 & 0,341 & 0,877 \\
\hline RI_08 & 3,33 & 0,747 & 0,562 & 0,871 \\
\hline RI_09 & 3,53 & 0,573 & 0,6 & 0,871 \\
\hline RI_10 & 3,15 & 0,731 & 0,513 & 0,872 \\
\hline RI_11 & 3,4 & 0,683 & 0,617 & 0,87 \\
\hline RI_12 & 3,49 & 0,635 & 0,69 & 0,868 \\
\hline RI_13 & 3,49 & 0,605 & 0,72 & 0,868 \\
\hline RI_14 & 2,31 & 1,103 & 0,524 & 0,872 \\
\hline RI_15 & 3,4 & 0,596 & 0,586 & 0,871 \\
\hline RI_16 & 2,89 & 0,916 & 0,539 & 0,871 \\
\hline RI_17 & 2,44 & 0,996 & 0,521 & 0,872 \\
\hline RI_18 & 2,6 & 1,011 & 0,423 & 0,876 \\
\hline RI_19 & 3,18 & 0,722 & 0,727 & 0,866 \\
\hline RI_20 & 2,16 & 1,316 & 0,373 & 0,882 \\
\hline
\end{tabular}

Fuente: elaboración propia. 
En la Figura I se presentan los $I F$ y los $I H c$ de los ítems. Podemos observar como todos los IHc>, 200, por lo que el Io०\% de los ítems se pueden considerar adecuados.

Respecto a los ID de los ítems, los más próximos a las dificultades medias tienen mayor variabilidad (es decir, discriminan mejor entre personas), mientras los más extremos tienen menor variabilidad. En este sentido el ítem que obtiene las puntuaciones más bajas es el RI_2O ('Dispone de recursos que facilitan el contacto con sus seres queridos') $(I D=2, \mathrm{I} 6)$. Por otro lado, el ítem que obtiene las puntuaciones más altas es el RI_o7 ('Mantiene el contacto que desea con su familia') $(I D=3,67)$. Estos resultados se representan en la Figura 2.

\subsection{Procedimiento}

La selección de la muestra se realizó mediante muestreo de conveniencia. En primer lugar, se envió un correo electrónico explicando los objetivos del estudio y solicitando la participación de los centros que atienden a personas con discapacidad de las distintas comunidades autónomas del territorio español. Seguidamente se envió un nuevo correo electrónico a aquellas entidades que mostraron su interés en participar con información acerca del proyecto de investigación, un enlace para inscribirse y poder acceder a la escala y al manual de instrucciones. También se facilitó un número de teléfono y una dirección de correo electrónico para que pudiesen realizar las consultas, comentarios o sugerencias que consideraran oportunas. Finalmente se negoció con cada entidad un plazo para completar las escalas en línea. El periodo de aplicación duró desde octubre de 2013 hasta la primera semana de febrero de 2014.

El análisis de resultados se llevó a cabo a través del paquete estadístico SPSS I9.0 para Windows.

\section{Resultados}

\subsection{Descriptivos de las puntuaciones en RI}

La distribución de las puntuaciones obtenidas en la subescala RI muestra una ligera asimetría positiva (asimetría $=, 076 ;$ curtosis $=-, 975$ ). Cada uno los 20 ítems se puntúan de I a 4 , de forma que la puntuación mínima posible es 20 y la máxima 80 . En este sentido, el rango de las puntuaciones oscila entre 45 y 80 , la media alcanza una puntuación de 60,4 $(D T=9,2)$ y la mediana un valor de 6I. Ambos valores superan ampliamente el punto medio teórico de la subescala (Punto Medio Teórico $=40$ ). De hecho, este valor es ya superado por el percentil 25. Asimismo, la puntuación alcanzada con mayor frecuencia por los participantes es $60(n=49 ; \mathrm{I}, 87 \%)$.

Si analizamos los porcentajes válidos de respuestas dadas por los participantes a cada uno de los ítems, encontramos que las respuestas más positivas ('siempre o casi siempre' en los ítems con valencia positiva) se obtienen en los ítems: RI_7 ('Mantiene el contacto que desea con su familia') ya que el 70,9\% responde 'siempre o casi siempre', mientras que ninguno selecciona la opción 'nunca o casi nunca'. El resto de ítems que presentaron mayores porcentajes de respuestas más positivas (entre el 56,4\% y el 50,9\%) fueron los siguientes: RI_9 ('Se ha identificado la mejor forma de comunicarle información (i.e., visual, táctil, auditiva, olfativa, gustativa)'); RI_I3 ('Se toman medidas específicas para mejorar sus habilidades de comunicación'); RI_I2 ('Las personas que le proporcionan apoyos comprueban si les entiende correctamente mediante el análisis de sus reacciones'); RI_2 ('Celebra acontecimientos importantes para él/ella y sus personas significativas (p. ej., cumpleaños, aniversarios)') y el ítem RI_I I ('Cuando se interactúa con éllella, se le proporciona el tiempo necesario para que pueda responder'). Entre el 9, I \% y el $47,3 \%$ responden con la categoría positiva máxima en cada uno de los catorce ítems restantes, donde los porcentajes se encuentran más repartidos entre las categorías. Estos resultados se muestran en la Figura 3. 


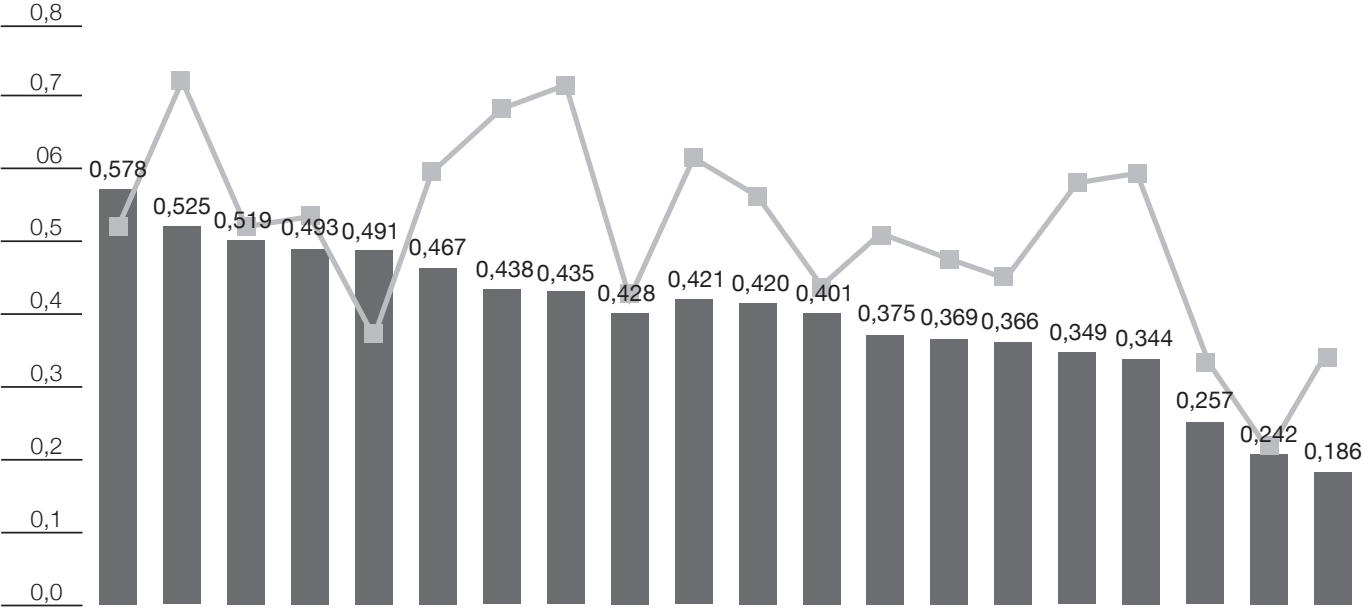

RI_14 RI_19 RI_17RI_16 R_20 RI_02 RI_12 Rl_13 RI_18 RI_11 Rl_08 RI_05 RI_10 Rl_03 RI_06 RI_15 Rl_09 RI_01 RI_04 RI_07

IF

Inc

Fuente: elaboración propia.

\section{Figura 2. Índices de dificultad de los ítems de la dimensión RI}

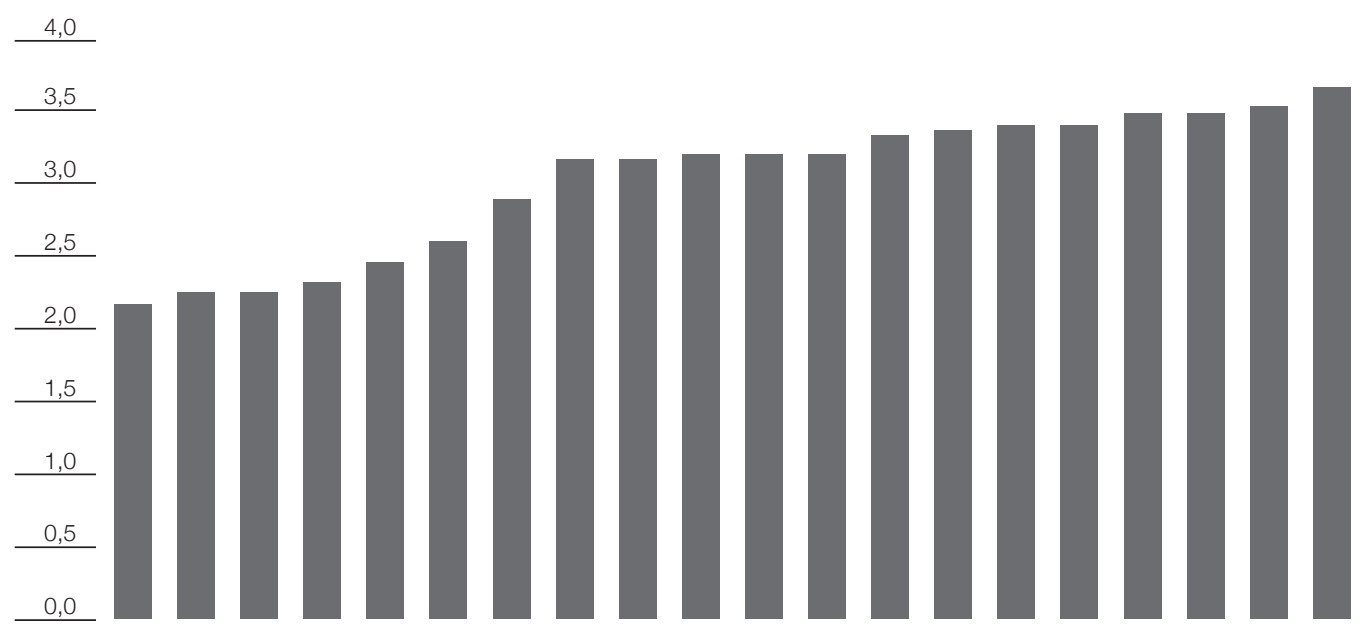

RI-20 RI-04 Rl-05 Rl-14 Rl-17 RI-18 Rl-16 Rl-10 RI-06 RI-01 Rl-03 Rl-19 RI-08 Rl-02 Rl-11 Rl-15 Rl-12 Rl-13 Rl-09 RI-07

Fuente: elaboración propia. 
En el otro extremo, las respuestas más negativas ('nunca o casi nunca') se obtienen en el ítem RI_2o ('Dispone de recursos que facilitan el contacto con sus seres queridos (p. ej., teléfono móvil, ayudas técnicas, internet'), al que el $50,9 \%$ responde 'nunca o casi nunca'. El resto de ítems que presentaron porcentajes de respuestas más negativas (entre el I 6,4 \% y el 29, I \%) fueron los ítems RI_4 ('Tiene oportunidades para estar a solas con sus amistades y personas conocidas), RI_I4 ('Tiene oportunidades para iniciar una relación de amistad si lo desea), RI_5 ('Se toman medidas especificas para mantener y extender sus relaciones sociales') y RI_I7 ('Utiliza un sistema de comunicación entendible en diferentes contextos).

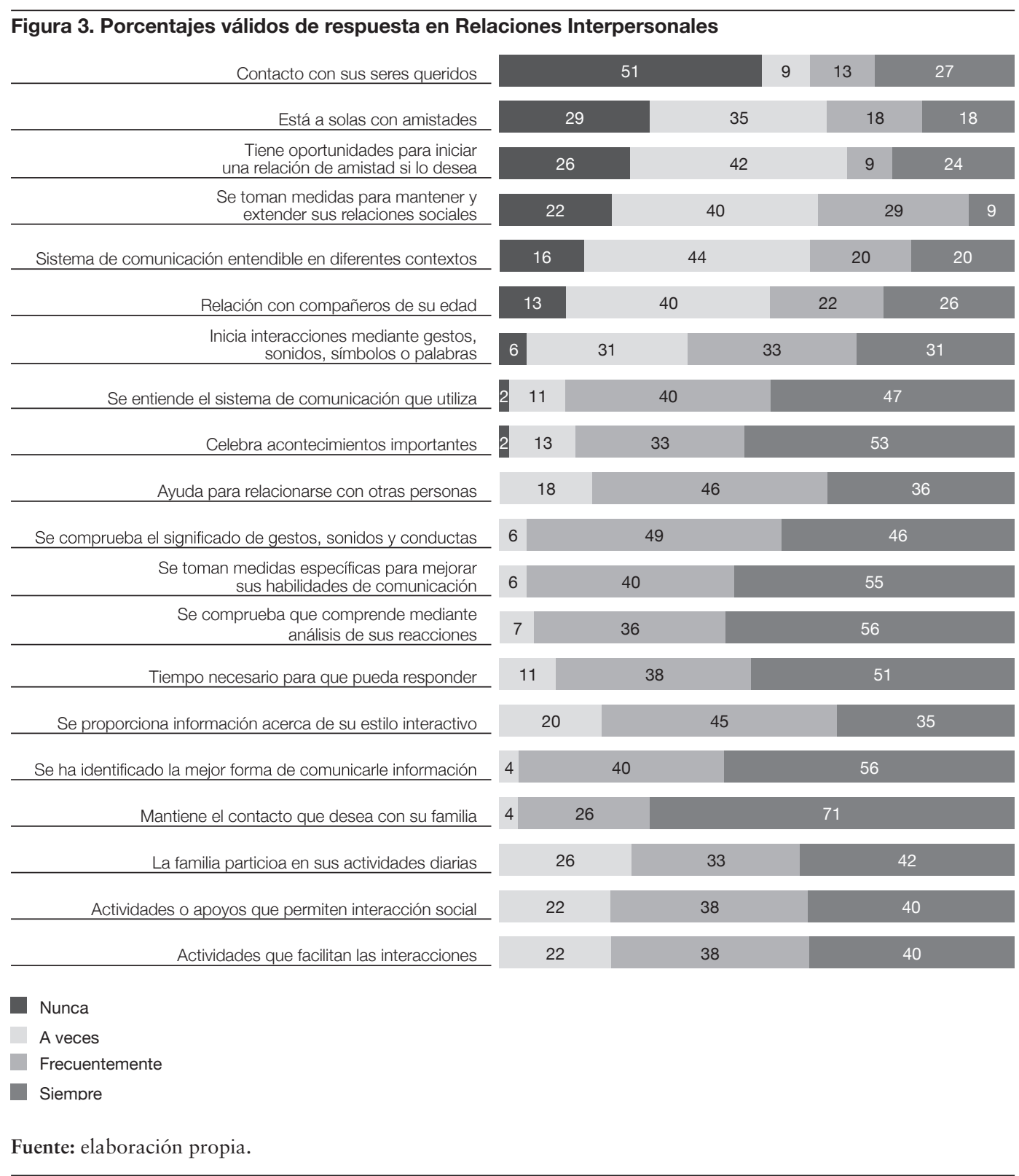




\subsection{Diferencias por variables sociodemográficas}

Para contrastar la relación entre la puntuación total obtenida en la subescala RI con la variable relativa al género, se ha utilizado la prueba $t$ para muestras independientes. Esta no dio lugar a diferencias significativas $\left.(t)_{53}\right)=-2, \mathrm{I}$; $p=, 8)$ : las puntuaciones fueron algo más positivas entre los varones $(M=60,6 ; D T=$ $9,8)$ que entre las mujeres $(M=60 ; D T=$ $7,8)$. Tampoco se encontraron diferencias significativas en función del género en ninguno de los 20 ítems.

En cuanto a las puntuaciones obtenidas por los participantes en la subescala RI en función de su $e d a d$, si atendemos a las medias observamos que son los participantes de 4 a 8 años los que obtienen el valor más alto $(M=63,4 ; D T=8,4)$, seguidos por los participantes entre 9 y I 2 años $(M=60,5 ; D T=9,2)$, los participantes entre I7 y 20 años $(M=60,6 ; D T=9,3)$ y, finalmente, con la puntuación más baja encontramos a los participantes entre I3 y I 6 años $(M=58,5$; $D T=9,8)$.

Los resultados del análisis de varianza (ANOVA) en la puntuación total de la dimensión RI no dieron lugar a diferencias significativas entre los grupos $\left(F_{(3)}=, 7 ; p=, 6\right)$. El mismo procedimiento se llevó a cabo para comprobar si existían diferencias por edad en cada uno de los veinte ítems que componen la dimensión RI. En dos de los ítems se encontraron diferencias estadísticamente significativas: RI_O5 ('Se toman medidas especificas para mantener $y$ extender sus relaciones sociales') $\left(F_{(3)}=5,5\right.$; $p=, 002 ; \eta^{2}=, 245$ ) y en el ítem RI_I4 ('Tiene oportunidades para iniciar una relación de amistad si lo desea') $\left(F_{(3)}=3, \mathrm{I} 25 ; p=, 034\right.$; $\eta^{2}=$, I 55 ).

Los resultados de las pruebas post hoc por ítems diferenciaron dos grupos en el ítem RI_05, así los participantes entre 4 y 8 años alcanzaron puntuaciones significativamente más altas que los participantes entre 9 y I 2 años $(p=, 029)$ y también mayores que los participantes entre I 3 y I 6 años $(p=, 005)$. En el ítem RI_I 4 la prueba $F$ de Scheffé no dio lugar a diferencias estadísticamente significativas.

Respecto a la puntuación total en la dimensión RI en función del tipo de escolarización, si atendemos a las medias observamos que son los participantes de educación combinada los que obtienen el valor más alto $(M=69,5$; $D T=8,3)$, seguidos de aquellos pertenecientes a la escolarización ordinaria $(M=62,7$; $D T=9)$ y, finalmente, con la puntuación más baja encontramos a los participantes de educación especial $(M=59,2 ; D T=8,99)$. Los resultados del ANOVA en la puntuación total de la dimensión RI no dieron lugar a diferencias significativas en función del tipo de escolarización $\left(F_{(2)}=2,660 ; p=, 079\right)$. El mismo procedimiento se llevó a cabo, posteriormente, para comprobar si existían diferencias por tipo de escolarización en cada uno de los veinte ítems que componen la dimensión RI. En tres de los ítems se encontraron diferencias estadísticamente significativas: en el ítem RI_05 ('Se toman medidas especificas para mantener $y$ extender sus relaciones sociales') $\left(F_{(2)}=5, \mathrm{IO}_{3}\right.$; $\left.p=, 009 ; \eta^{2}=, \mathrm{I} 64\right)$ ); en el ítem RI_I4 ('Tiene oportunidades para iniciar una relación de amistad si lo desea') $\left(F_{(2)}=3,67 \mathrm{I} ; p=, 032\right.$; $\eta^{2}=$, I 24) $y$ en el ítem RI_I 5 ('Se comprueba de forma sistemática el significado de sus gestos, sonidos y conductas') $\left(F_{(2)}=4, \mathrm{I} ; p=, 022\right.$; $\left.\eta^{2}=, \mathrm{I} 36\right)$.

Los resultados de las pruebas post hoc por ítems diferenciaron un grupo en el ítem RI_I4 y dos grupos en los ítems RI_05 y RI_I 5. En el ítem RI_05 los participantes de educación combinada alcanzaron puntuaciones significativamente más altas que los participantes de educación especial $(p=$, OI I $)$. En el ítem RI_I 5 las diferencias significativas se dieron también entre estos dos mismos grupos $(p=, \infty \circ)$.

El análisis de la puntuación total en la subescala RI según el nivel de DI (leve, moderada, severa o profunda) revela, en primer lugar, la diferencia en el número de participantes incluidos en cada categoría. Así encontramos que el grupo más numeroso es el conformado por personas con DI severa $(n=37)$, seguido del de personas con DI 
moderada $(n=\mathrm{II})$, y del grupo de personas con DI profunda $(n=6)$. Finalmente el nivel leve de DI es el menos numeroso de todos $(n=\mathrm{I})$. Puesto que dicho nivel cuenta con un solo participante no podemos realizar pruebas post hoc, por lo que se optó por eliminar esta categoría. De este modo, son los participantes con DI moderada los que obtienen el valor más alto $(M=65,9$; $D T=8,5)$, seguidos por los participantes con DI severa $(M=60,5 ; D T=8,7) \mathrm{y}$, finalmente, aquellos con DI profunda $(M=50,6 ; D T=6,6)$. El ANOVA puso de manifiesto la existencia de diferencias significativas en función del nivel de DI $\left(F_{(6)}=10,85 ; p=, 004 ; \eta^{2}=\right.$, I 98$)$ y la prueba de comparaciones múltiples constató la existencia de dos grupos. Las personas con DI profunda obtuvieron puntuaciones significativamente más bajas que aquellos con DI severa y aquellos con DI moderada.

También se encontraron diferencias por el nivel de DI en siete de los ítems. Los resultados de las pruebas post hoc por ítems diferenciaron dos grupos en cada uno de los ítems, excepto en el ítem RI_I4 ('Tiene oportunidades para iniciar una relación de amistad si lo desea') en el que no aparecieron diferencias estadísticamente significativas. En el ítem RI_O2 ('Celebra acontecimientos importantes para éll ella y sus personas significativas') las personas con DI moderada alcanzaron puntuaciones significativamente más altas que las personas con DI profunda $(p=, 039)$. En el ítem RI_o8 ('Las personas que le proporcionan apoyos entienden el sistema de comunicación que utiliza'), los participantes con DI profunda años alcanzaron puntuaciones significativamente más bajas que los participantes con DI severa ( $p=$, oI 9$)$ y que los participantes con DI moderada ( $p=$,oI9). En el ítem RI_I I ('Cuando se interactúa con éllella, se le proporciona el tiempo necesario para que pueda responder') los participantes con DI severa alcanzaron puntuaciones significativamente más altas que los participantes con DI profunda ( $p=$, o I 7 ). En el ítem RI_I 2 ('Las personas que le proporcionan apoyos comprueban si les entiende correctamente mediante el análisis de sus reacciones') las diferencias significativas se dieron también entre estos dos mismos grupos $(p=, 022)$ a favor del grupo con DI severa. A su vez, en el ítem RI_I6 ('Inicia interacciones mediante gestos, sonidos, simbolos o palabras') las personas con DI moderada alcanzaron puntuaciones significativamente más altas que las personas con DI profunda $(p=, 003)$.En el ítem RI_I7 ('Utiliza un sistema de comunicación entendible en diferentes contextos') los participantes con DI moderada alcanzaron puntuaciones significativamente más altas que los participantes con DI severa $(p=$ ,035) y profunda $(p=, 003)$. En el caso del ítem RI_20 ('Dispone de recursos que facilitan el contacto con sus seres queridos') $\left(F_{(2)}=6,907 ; p=\right.$ ,००2; $\eta^{2}=, 2 \mathrm{I} 3$ ) las personas con DI moderada alcanzaron puntuaciones significativamente más altas que las personas con DI severa $(p=, 002)$.

Para contrastar la relación entre la puntuación total obtenida en la subescala RI con la variable relativa a la presencia o no de problemas de comportamiento, utilizamos la prueba $t$ para muestras independientes. Ésta no dio lugar a diferencias significativas $\left(t\left(_{53}\right)=\mathrm{I}, 540\right.$; $p=, \mathrm{I} 29)$ las puntuaciones fueron algo más positivas entre los participantes sin problemas de comportamiento $(M=6 \mathrm{I}, 8 ; D T=9,6)$ que entre los que sí presentaban problemas de comportamiento $(M=57,7 ; D T=7,9)$. En cuanto a los ítems (Figura 4), sólo se encontraron diferencias significativas en el ítem RI_20 ('Dispone de recursos que facilitan el contacto con sus seres queridos').

\section{Discusión y conclusiones}

El objetivo general que ha guiado este trabajo ha consistido en analizar de forma exhaustiva las RI de los niños y jóvenes con TEA y DI. La distribución en la puntuación de la subescala RI resultó asimétrica negativa. Asimismo, la media, la moda y la mediana superaron el punto medio teórico de la escala RI. Por tanto, si bien las puntuaciones de niños y jóvenes con TEA en RI son positivas (por encima del punto medio teórico de la subescala), las puntuaciones 


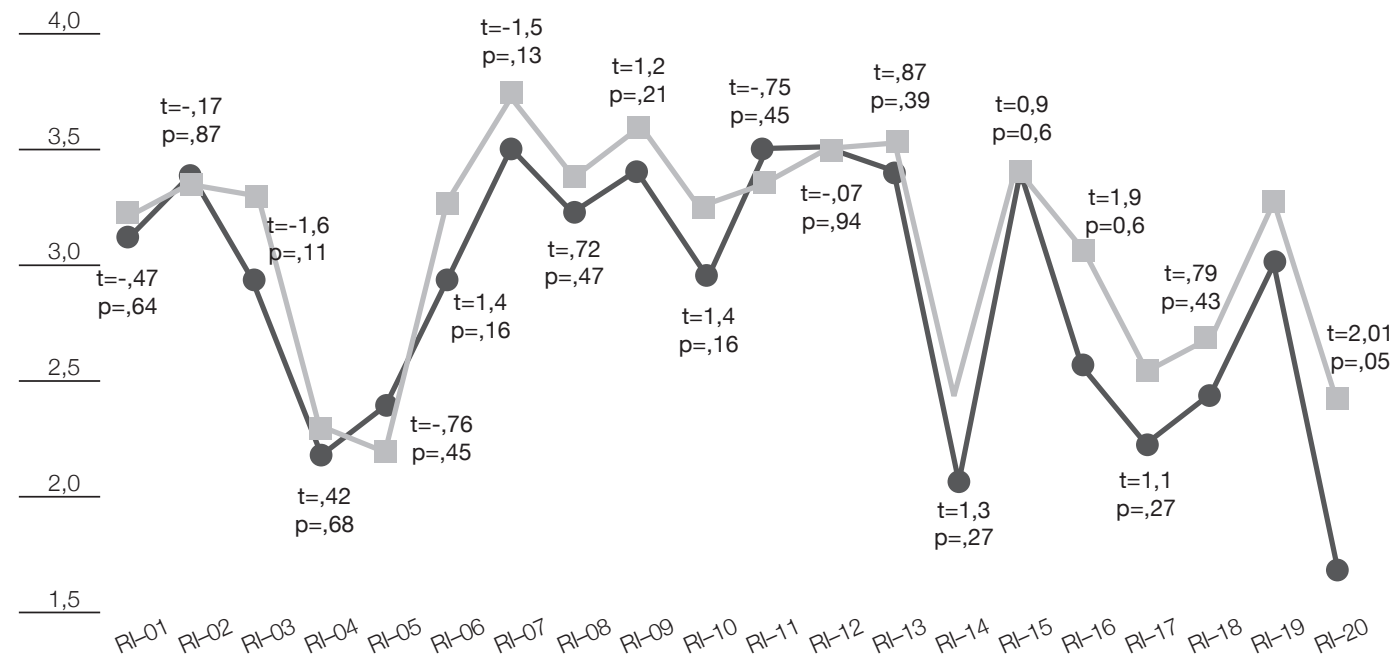

Sí

No

Fuente: elaboración propia.

son mejorables y se detectan necesidades importantes.

Respecto a los ítems, los participantes obtuvieron las mejores puntuaciones en el ítem RI_7 ('Mantiene el contacto que desea con su familia'), lo que no resulta sorprendente si tenemos en cuenta que el $72,73 \%$ de los participantes residen en el hogar familiar. También encontramos altas puntuaciones en algunos de los ítems relativos a la comunicación. En este sentido, parece que los esfuerzos y las prácticas por parte de las organizaciones destinadas a solventar los problemas de comunicación (uno de los rasgos centrales del TEA) son exitosos.

En cambio, las peores puntuaciones se obtuvieron en el ítem RI_2o ('Dispone de recursos que facilitan el contacto con sus seres queridos (p. ej., teléfono móvil, ayudas técnicas, internet'), lo que puede interpretarse como la no necesidad de tales recursos dado que la mayor parte de los participantes viven con sus familias.
También se obtuvieron bajas puntuaciones en los ítems relativos a fomentar las interacciones sociales. Estos resultados reflejan las dificultades que estos niños y jóvenes presentan a la hora de establecer contactos y relaciones sociales, lo que a su vez pone en entredicho la eficacia de los programas centrados en este ámbito, tal vez por no estar ajustados a las verdaderas necesidades de las personas (Walton e Ingersoll, 2013: 595). En cuanto al género, si bien la muestra de participantes con TEA estaba formada en su mayoría por varones $(72,7 \%)$, lo que se corresponde con la media de distribución del sexo en los TEA de una mujer por cada cuatro varones (Lee et al., 2008: I I 50), no se observan diferencias significativas, lo que concuerda con los resultados obtenidos en anteriores investigaciones (Gómez, 2010: 659).

Respecto a la edad aunque los análisis de varianza no dieron lugar a diferencias significativas en la puntuación total en RI, si atendemos a las medias los participantes de 4 a 8 años obtienen el valor más alto, seguidos 
por los participantes entre 9 y I 2 años. Por su parte, los jóvenes entre I 3 y I 6 años obtienen la puntuación más baja y, si bien se produce un aumento de las puntuaciones entre aquellos de edades comprendidas entre 17 y 20 años, no alcanzan los niveles encontrados en los participantes entre 4 y 8 años. El hecho de que no aparezcan diferencias significativas es una evidencia a favor de que la subescala RI puede ser válida para todas las edades. Sin embargo, sí aparecieron diferencias significativas en función de la edad en el ítem RI_05 'Se toman medidas especificas para mantener y extender sus redes sociales'; así ,los participantes entre 4 y 8 años alcanzaron puntuaciones significativamente más altas que aquellos de edades entre 9 y I 2 años y también mayores que los participantes entre I3 y I 6 años. Estos resultados concuerdan con los hallados en la literatura, en los que los padres de los niños con TEA de menor edad informan de mayores puntuaciones en la CV que los padres de los niños de mayor edad. Resulta pues importante conocer los factores que pueden influir en el descenso de las puntuaciones al aumentar la edad del niño, ya que permitirían identificar y poner en marcha estrategias de intervención eficaces (Kuhlthau et al., 2010: 727), lo que sería posible a través de un estudio longitudinal.

El análisis de la puntuación total en la dimensión RI en función del tipo de escolarización revela que la gran mayoría de los participantes $(80 \%)$ pertenecen al grupo de educación especial. Si bien los resultados del ANOVA en la puntuación total de esta dimensión no dieron lugar a diferencias significativas, si atendemos a las medias observamos que los participantes de educación combinada obtienen el valor más alto, seguidos por aquellos pertenecientes a la escolarización ordinaria y, finalmente, con la puntuación más baja encontramos a los participantes de educación especial. Además, en dos de los ítems, los participantes de educación combinada alcanzaron puntuaciones significativamente más altas que los participantes de educación especial. Estos resultados reflejan como la educación inclusiva mejora las RI y, por tanto, la CV de los niños y adolescentes con TEA. Sin embargo, también reflejan la dificultad para poner en práctica dicha inclusión en las escuelas ordinarias. Todo ello apunta a la necesidad de cambios profundos en la cultura de los centros, y así conseguir el mayor desarrollo posible de las capacidades personales, sociales e intelectuales de todos los alumnos, sin discriminación alguna (Gutiérrez, 2006: xiii).

Se encontraron diferencias significativas también en la puntuación total en RI en función del nivel de DI. Las personas con DI profunda obtuvieron puntuaciones significativamente más bajas que aquellos con DI severa y moderada. Asimismo, se encontraron diferencias estadísticamente significativas en ocho de los ítems. Estos resultados concuerdan con la bibliografía analizada, según la cual los niños con TEA que presentan déficits sociales obtienen menores puntuaciones en desarrollo cognitivo (Gillis et al., 20I I: 352). A pesar de ello, si bien es cierto que las investigaciones han demostrado que las personas con TEA de todos los niveles de funcionamiento intelectual pueden beneficiarse de intervenciones centradas en el aprendizaje de habilidades sociales que mejoran sus interacciones sociales (Walton e Ingersoll, 20I3: 6I2), estos hallazgos ponen de manifiesto que las personas con DI severa y profunda han sido el colectivo que ha disfrutado de estos avances en menor medida y para quienes la implementación de estos cambios ha resultado más compleja (Gómez et al., en prensa). Por tanto, las personas con TEA y DI severa y profunda presentan otro tipo de necesidades que no pueden ser cubiertas por los programas diseñados para las personas con TEA y DI moderada (Walton e Ingersoll, 20I3: 595), sino que deben ser específicos, es decir ajustados al nivel de funcionamiento que presenta la persona.

Respecto a la presencia o no de problemas de comportamiento, cabe destacar la alta prevalencia de problemas de comportamientos en la muestra $(32,7 \%)$, lo que corresponde con los hallazgos de la literatura especializada que señalan que una de cada tres personas con TEA presenta problemas de comportamiento (Emerson, 2003: 56). Si bien no aparecen diferencias significativas en la puntuación total 
en RI en función de la presencia de problemas de comportamiento, las puntuaciones fueron más positivas entre los participantes que no los presentan. Estos datos sugieren que las intervenciones dirigidas a la reducción y/o eliminación de dichos comportamientos pueden mejorar la CV tanto de las personas con TEA como la de sus familias (Lee et al., 2008: I I 59).

Los resultados de este estudio tienen importantes implicaciones en la intervención sobre las necesidades en las RI de niños y adolescentes con TEA. El nivel de habilidades sociales a lo largo de la vida es un importante predictor del bienestar psicológico. Por este motivo, la intervención temprana desde el momento en que las alteraciones en las RI son detectadas es fundamental para incrementar el funcionamiento social del niño y, por tanto, mejorar su CV (Tureck y Matson, 20I 2: 6I3). No obstante este estudio no está libre de limitaciones y entre ellas cabe destacar las características de la muestra (número reducido de participantes no seleccionados al azar), por lo que sus conclusiones no pueden generalizarse. Otro de sus puntos débiles es que si bien los informes de otras personas han sido utilizados casi en exclusiva para evaluar la CV de personas con TEA y reúnen los criterios de validez, han mostrado una baja-media correlación con los autoinformes de los niños con TEA, siendo estos últimos considerados como la mejor forma de evaluar la CV.

Con respecto a las líneas futuras de investigación, es mucho aun lo que desconocemos acerca de cómo las RI determinan la CV calidad de estas personas. Es imprescindible por tanto continuar con el estudio y análisis de las necesidades de los niños con TEA y las de sus familias, de forma que permitan la puesta en marcha de los servicios y apoyos necesarios para una vida de calidad. En este sentido este estudio constituye una primera aproximación a la evaluación de la CV de niños y jóvenes con TEA y DI, que facilita la implementación de prácticas basadas en la evidencia y el desarrollo de planes individuales de apoyo.

\section{Agradecimientos}

El equipo de investigación ha trabajado en el marco del proyecto de investigación del Ministerio de Economía y Competitividad PSI20I 2-33 I39. Los autores quisieran agradecer a las organizaciones, personas con discapacidad intelectual, profesionales y familiares el haber completado los cuestionarios. 
Referencias bibliográficas

American Psychiatric Association. (2013): Diagnostic and statistical manual of mental disorders ( $5^{\text {th }}$ ed.), Washington, D.C.: American Psychiatric Association.

Cuesta, J. L. (2009): Trastornos del espectro autista y calidad de vida. Guía de indicadores para evaluar organizaciones y servicios, Madrid: La Muralla.

Deckers, A. et al. (20I4): "Desire for social interaction in children with autism spectrum Disorders”. Research in Autism Spectrum Disorders, 8, 449-453.

Emerson, E. (2003): "Prevalence of psychiatric disorders in children and adolescents with and without intellectual disability". Journal of Intellectual Disability Research, 47, 5I-58.

España. Resolución de 2 de diciembre de 2008 , por la que se establecen criterios comunes de acreditación para garantizar la calidad de los centros y servicios del Sistema para la Autonomía y Atención a la Dependencia. Boletín Oficial del Estado, 17 de diciembre de 2008, núm. 303, pp. 50722-50725.

España. Ley 39/2006, de I4 de diciembre, de Promoción de la Autonomía Personal y Atención a las personas en situación de dependencia. Boletín Oficial del Estado, I 5 de diciembre de 2006, núm. 299, pp. 44 I4244 I 56.

Gillis, J. M. et al. (20I I): “Assessment of social behavior in children with autism: The development of the Behavioral Assessment of Social Interactions in Young Children". Research in Autism Spectrum Disorders, 5:35 I360.

Gómez, L. E. (2010):“Evaluación de la calidad de vida en servicios sociales: validación y calibración de la escala GENCAT" (Tesis doctoral) (en línea). http://gredos.usal.es/jspui/ handle/ı0366/76489', acceso 5 de mayo de 2014 .

Gómez, L. E. et al. (en prensa): "Escala KidsLife”. Salamanca: INICO.
Gómez, L. E. et al. (20I0a):“Calidad de vida individual: avances en su conceptualización y retos emergentes en el ámbito de la discapacidad". Behavioral Psychology/ Psicología Conductual, I 8(3): 453-472.

Gómez, L. E. et al. (20IOb): “A comparison of alternative models of individual quality of life". Social IndicatorsResearch, Iог: I09-I 26.

Gómez, L. E. et al. (2014): "El constructo de calidad de vida en niños y adolescentes con discapacidades múltiples y profundas: propuesta para su evaluación”. Siglo Cero, 45 (I): 56-69.

Gutiérrez, I. (2006): "Prólogo" en Aranda, R. E. (coord.):Educación especial: áreas curriculares para alumnos con necesidades educativas especiales, Madrid: Pearson-Prentice Hall.

Kuhlthau, K. et al. (2010): "Health-Related Quality of Life in Children with Autism Spectrum Disorders: Results from the Autism Treatment Network". Journal of Autism and Developmental Disorders, 40:72 I-729.

Lee, L. et al. (2008):“Children with Autism: Quality of Life and Parental Concerns. Journal of Autism and Developmental Disorders", 38 : I I 47-I I 60.

Schalock, R. L. et al. (2009): Quality of life for people with intellectual and other developmental disabilities. Applications across individuals, organizations, communities, and systems, Washington, DC: American Association on Intellectual and Developmental Disabilities (Orig. 2007).

Schalock, R. L. et al. (20Iо): "Quality of life model development in the field of intellectual disability, en R. Kober (dir.): Quality of life for people with intellectual disability. Nueva York: Springer.

Schalock, R. L. y Verdugo, M. A. (2003): Quality of life for human service practitioners. Washington, DC: American Association on Mental Retardation. (Orig. 2002). 
Schalock, R. L. y Verdugo, M. A. (2007):“El concepto de calidad de vida en los servicios y apoyos para personas con discapacidad intelectual”. Siglo Cero, 38:2 I-36.

Schalock, R. L. y Verdugo, M. A. (2012):“A conceptual and measurement framework to guide policy development and systems change". Journal of Policy and Practice in Intellectual Disabilities, 7: 7I-8I.

Sheldrick, R. C. et al. (20I2): "Quality of life of adolescents with autism spectrum disorders: concordance among adolescents' self-reports, parents' reports, and parents' proxy reports". Quality of Life Research, 2I: 53-57.

Summers, J. A. et al. (2005): “Conceptualizing and measuring family quality of life". Journal of Intellectual Disability Research, 49: 777-783.

Tureck, K. y Matson, J. L. (2OI 2): “An examination of the relationship between autism spectrum disorder, intellectual functioning, and social skills in children". Journal of Developmental and Physical Disabilities, 24:607-6I 5.

Verdugo, M. A. et al. (2007):“Construcción de escalas de calidad de vida multidimensionales centradas en el contexto: La escala Gencat”. Siglo Cero, 38(4): 57-72.

Walton, K. M. e Ingersoll, B. R. (2OI3): “Improving social skills in adolescents and adults with autism and severe to profound intellectual disability: a review of the literature". Journal of Autism and Developmental Disorders, 43: 594-61 5 .

WHOQOL Group (1998): “The World Health Organization WHOQOL-BREF quality of life assessment”. Psychological Medicine, 28: $55 \mathrm{I}-55^{8}$. 